\title{
Acompanhamento de pacientes do espectro esquizofrênico no território: adaptação da intervenção para períodos de transição ("Critical Time Intervention") para o contexto brasileiro em Centros de Atenção Psicossocial do município do Rio de Janeiro
}

Monitoring of patients within the schizophrenia spectrum: adaptation of intervention for transition periods ("Critical Time Intervention") for the Brazilian context in Psychosocial Care Centers in the city of Rio de Janeiro

Flávia Mitkiewicz de Souza1', Maria Cecília de Araújo Carvalho², Elie Valência ${ }^{3}$, Tatiana Fernandes Carpinteiro da Silva ${ }^{4}$, Catarina Magalhães Dahl${ }^{5}$, Maria Tavares Cavalcanti ${ }^{6}$

\begin{abstract}
Resumo
A continuidade do cuidado de pacientes com transtornos mentais severos e persistentes depende da facilidade de acesso aos serviços. A noção de continuidade do cuidado implica também que os serviços sejam abrangentes, indicando a existência dos destinados à intervenção em crises; de residências terapêuticas; de diferentes dispositivos de tratamento; oportunidades de lazer e redes de suporte social; e oferta de transporte para o acesso físico aos serviços oferecidos. Estudos mostram que a boa continuidade de cuidados associa-se à melhor qualidade de vida, melhor funcionamento social, menor gravidade de sintomas e maior satisfação com o serviço. Neste artigo são apresentados os resultados do estudo piloto que visou adaptar para o contexto brasileiro a Critical Time Intervention (CTI) e testar sua viabilidade com pessoas com transtornos do espetro esquizofrênico em tratamento nos Centros de Atenção Psicossocial (CAPS) do município do Rio de Janeiro. O desenho da pesquisa incluiu três fases inter-relacionadas. O objetivo deste artigo é descrever o trabalho realizado na Fase 3 do estudo - implementação da intervenção - por meio dos resultados do acompanhamento de 20 pacientes esquizofrênicos em tratamento nos 4 CAPS do município do Rio de Janeiro a partir da análise dos focos definidos pela intervenção (CTI-Br)
\end{abstract}

Palavras-chave: serviços de saúde mental; esquizofrenia; continuidade da assistência ao paciente.

Trabalho realizado no Instituto de Psiquiatria da Universidade Federal do Rio de Janeiro (UFRJ) - Rio de Janeiro (RJ), Brasil.

${ }^{1}$ Mestranda do Instituto de Psiquiatria da Universidade Federal do Rio de Janeiro (UFRJ) - Rio de Janeiro (RJ), Brasil.

2Professora da Escola Politécnica de Saúde Joaquim Venancio da Fundação Oswaldo Cruz (FIOCRUZ) - Rio de Janeiro (RJ), Brasil.

${ }_{3}^{3}$ Professor Assistente do Departamento de Epidemiologia de Saúde Pública da Escola Mailman da Universidade de Columbia - Nova Iorque, Estados Unidos.

${ }^{4}$ Doutoranda do Instituto de Estudos em Saúde Coletiva da UFRJ - Rio de Janeiro (RJ), Brasil.

${ }^{5}$ Mestre em Saúde Coletiva pela Universidade Estadual de Campinas (UNICAMP) - Campinas (SP), Brasil.

${ }^{6}$ Diretora do Instituto de Psiquiatria da UFRJ - Rio de Janeiro (RJ), Brasil.

Endereço para correspondência: Flávia Mitkiewicz de Souza - Rua Almirante Alexandrino, 1.109 - Santa Teresa - CEP: 20241-260 - Rio de Janeiro (RJ), Brasil -

E-mail: fmitkiewicz@terra.com.br.

Fonte de financiamento: Conselho Nacional de Desenvolvimento Científico e Tecnológico (CNPq).

Conflito de interesse: nada a declarar. 


\begin{abstract}
The continuity of care for patients with severe and persistent mental disorders depends on the ease of access to services. The notion of continuity of care also implies that services indicate existence of comprehensive services for crisis intervention, residential treatment, different treatment devices, leisure opportunities and social support networks, and that they offer transportation for physical access services offered. Studies show that good continuity of care is associated with better quality of life, better social functioning, lower severity of symptoms and greater satisfaction with services. This article presents the results of the pilot study that aimed to adapt "Critical Time Intervention" (CTI) to the Brazilian context and tested its viability with people with schizophrenia spectrum disorders treated in Psychosocial Care Centers in the city of Rio de Janeiro. The research design included three interrelated phases. The aim of this paper is to describe the work done in Phase 3 implementation of the intervention - by analyzing the results of the monitoring of 20 schizophrenic patients in four Rio de Janeiro Psychosocial Care Centers as part of CTI-Br.
\end{abstract}

Keywords: mental health services; schizophrenia; continuity of patient care.

\section{INTRODUÇÃO}

A continuidade do cuidado deve ser pensada na atenção a todos os usuários do sistema de saúde, mas é um elemento que se torna mais expressivo, no que concerne a sua qualidade, quando ofertado aos pacientes que utilizam diferentes serviços ao longo da vida, tais como os portadores de transtornos mentais severos e persistentes. Esta continuidade é resultado da combinação de fatores como acesso adequado ao cuidado, habilidades comuns dos profissionais, bom fluxo de informação, entendimento entre técnicos e organizações e boa coordenação e integração entre profissionais para manter a consistência da atenção ${ }^{1}$.

A origem da discussão teórica e conceitual sobre continuidade de cuidados remete aos anos $1960^{2}$. Diferentes definições de continuidade foram reunidas de forma esquemática por alguns autores ${ }^{3}$, enfatizando o seu aspecto temporal ou longitudinal: a continuidade é uma relação entre cuidado passado e presente de acordo com as necessidades terapêuticas do paciente.

Para Test, é fundamental que durante todo o tratamento o paciente possa ter acesso ao cuidado que necessita, podendo vir a ocorrer descontinuidade, com abandono do tratamento, quando algum elemento essencial do cuidado não estiver presente ou quando existirem falhas na coordenação ou comunicação. Bachrach ${ }^{4}$ define continuidade de cuidados como um processo que envolve o movimento ininterrupto de pacientes entre os diversos elementos do sistema de prestação de serviços de saúde.

Para Bachrach (1986), a continuidade do tratamento do paciente com transtornos mentais severos e persistentes depende da facilidade de acesso aos serviços. São características importantes para a sua efetivação o bom acolhimento por parte das equipes, com condutas flexíveis, pois estes pacientes podem ter dificuldades para aprender as regras de circulação no sistema de cuidados, e disponibilidade para a prestação do cuidado por um longo tempo, em locais que sejam facilmente acessíveis.

A noção de continuidade de cuidados implica também que os serviços sejam abrangentes (o que correspondente à dimensão da integralidade), incluindo o cuidado em situações de intervenção em crises; de residências terapêuticas; diferentes dispositivos de tratamento; oportunidades de lazer e redes de suporte social; e oferta de transporte para o acesso físico aos equipamentos oferecidos. Estudos mostram que a boa continuidade de cuidados associa-se à melhor qualidade de vida, melhor funcionamento social, menor gravidade de sintomas e maior satisfação com o serviço ${ }^{5}$.

Entre 2009 e 2010, o Laboratório de Avaliação de Serviços e Qualidade de Vida em Saúde Mental do Instituto de Psiquiatria da Universidade Federal do Rio de Janeiro (UFRJ), com o apoio do Conselho Nacional de Desenvolvimento Científico e Tecnológico (CNPq - Edital 33/2008 - processo 575089/2008-3) e em parceria com Instituto de Estudos em Saúde Coletiva da UFRJ e o Departamento de Epidemiologia da Escola Mailman de Saúde Publica da Universidade de Columbia (Nova Iorque, Estados Unidos), desenvolveu um projeto de pesquisa intitulado: "Intervenção em saúde mental para períodos de transição - adaptação da intervenção no tempo crítico (Critical Time Intervention - CTI) para a realidade brasileira e estudo piloto em pacientes do espectro esquizofrênico atendidos nos Centros de Atenção Psicossocial (CAPS) do Rio de Janeiro". Este projeto visou adaptar e avaliar a viabilidade da implementação de um programa comunitário de saúde mental para pessoas com transtornos graves em situação de transição. Em situação de transição consideramos aquelas em que os usuários encontram-se suscetíveis à descontinuidade do tratamento, como: agravamento dos sintomas psiquiátricos; uso abusivo de álcool e outras drogas; estar em situação de rua; estar em processo de alta hospitalar e/ou desinstitucionalização; situações de desestruturação familiar e fazer uso irregular de medicação.

$\mathrm{O}$ desenho da pesquisa incluiu três fases inter-relacionadas. A primeira consistiu em trabalho de campo no qual utilizamos os métodos qualitativo e quantitativo. Esta etapa foi eminentemente exploratória e consistiu no mapeamento das características sociodemográficas, clínicas e de utilização de serviços dos usuários dos Centros de Atenção Psicossocial 
(CAPS), bem como na identificação das necessidades concretas dos participantes do estudo. No período de fevereiro a junho de 2009, pesquisadores realizaram entrevistas com usuários, familiares e trabalhadores e participaram da rotina de trabalho de quatro CAPS de uma área programática (AP) do Rio de Janeiro - AP 3. À época do trabalho de campo, existiam dez CAPS voltados para o atendimento de adultos e distribuídos em dez APs na cidade do Rio de Janeiro. Optamos por delimitar o campo de pesquisa à Zona Norte da cidade, abarcando as APs 3.1, 3.2 e 3.3.

A segunda fase consistiu na tradução do manual clínico da CTI para incluir as adaptações feitas para o seu uso no Brasil, oriundas de dados coletados na primeira fase, bem como no treinamento de profissionais de nível médio a fim de que atuassem como agenciadores da CTI-Br. De julho de 2009 a fevereiro de 2010 foi realizado o mapeamento das características sociodemográficas, clínicas, de utilização de serviços e de necessidades concretas e de saúde em uma amostra de 150 usuários com transtorno do espectro esquizofrênico, por meio da aplicação de entrevistas semiestruturadas (Questionário Central CTI-Br) e questionários e escalas estruturadas (Escala das Síndromes Positiva e Negativa - PANSS, do inglês Positive and Negative Syndrome Scale; entrevista diagnóstica - MiniInternational Neuropsychiatric Interview (MINIPLUS) e Escala de qualidade de vida - QLS-Br, do inglês Quality of Life Scale). Essas entrevistas foram aplicadas posteriormente em todos os usuários que participaram da intervenção na Fase 3, conforme protocolo de pesquisa.

No trabalho, realizado entre os meses de janeiro e março 2010, foram traduzidos para o português o manual clínico da CTI, o formulário de datas das fases, o formulário de supervisão, notas sobre o progresso da intervenção e nota de fechamento de caso da CTI-Br.

Seguindo a orientação do manual clínico, a coordenadora geral do projeto conduziu o processo de capacitação dos profissionais para atuarem como agenciadores de caso durante a implementação da intervenção.

A terceira fase consistiu na implementação da intervenção adaptada em um grupo de 20 pacientes com transtornos do espectro esquizofrênico.

O objetivo deste artigo é descrever o trabalho realizado na Fase 3 do estudo - implementação da intervenção - por meio dos resultados do acompanhamento de 20 pacientes esquizofrênicos em tratamento nos 4 CAPS do município do Rio de Janeiro a partir da análise dos focos definidos pela intervenção (CTI-Br), que serão posteriormente descritos. Para fins elucidativos, introduziremos uma breve apresentação sobre a CTI, nomeada na adaptação brasileira como Intervenção para Períodos de Transição (CTI-Br).

\section{Intervenção no tempo crítico}

A intervenção no tempo crítico foi originalmente criada em Nova Iorque, nos Estados Unidos, no início da década de 1990, a partir dos obstáculos encontrados para garantir a continuidade do tratamento às pessoas com o diagnóstico de esquizofrenia em situação de rua ${ }^{6}$. O planejamento de transição da população residente nos abrigos para inserção na comunidade falhava sistematicamente. As pessoas tinham dificuldade em permanecer nos locais de tratamento e moradia para os quais eram encaminhadas e retornavam para os albergues, no famoso fenômeno da porta giratória ${ }^{7,8}$, em semelhança ao que ocorre na psiquiatria, sendo este caracterizado pelas re-hospitalizações frequentes dos portadores de transtornos mentais, obedecendo a um ciclo recidivo de internação/alta/internação.

A CTI foi criada com o objetivo de complementar e fortalecer a rede de serviços de saúde e suporte social, auxiliando pessoas portadoras de transtornos do espectro esquizofrênico a se engajarem e estabilizarem em um cuidado continuado. Trata-se de um modelo inovador, desenvolvido inicialmente para ser administrado durante o período de transição de situações críticas, como hospitalização, encarceramento, situação de rua/albergamento e ausência ou precariedade de tratamento, visando à inserção em serviços de apoio e tratamento de longo prazo'.

Ela é uma intervenção com duração de tempo pré-determinada (em geral nove meses) constituída por dois componentes. O primeiro visa o estabelecimento de vínculos estáveis dos portadores de transtornos mentais severos e persistentes com os sistemas de apoio formal e informal, principalmente com as redes de saúde e suporte social e familiar, e o segundo centra-se no apoio emocional e prático durante o tempo crítico de transição. O trabalho, como vimos, concentra-se em áreas consideradas cruciais para a estabilização e a inserção social do paciente na comunidade, os chamados focos da intervenção, e é executado em três fases consecutivas: inicialmente os encontros entre agenciador e usuário são bastante frequentes, mas o programa é planejado de forma que os contatos entre os pares diminuam ao longo dos meses, buscando favorecer a adesão e a continuidade do cuidado e promover a autonomia do usuário ${ }^{10}$. A intervenção é executada por um grupo de agenciadores de caso (case management) e um coordenador clínico com o apoio de um psiquiatra ${ }^{11}$. No caso da adaptação brasileira, o psiquiatra faz parte da equipe do CAPS ao qual o paciente estava vinculado.

\section{METODOLOGIA}

A análise concentra-se no material obtido pelas notas de campo dos profissionais de nível médio que atuaram como 
agenciadores de caso na Fase 3 da pesquisa. Nela, um grupo de usuários dos CAPS recebeu a intervenção, por meio do acompanhamento de agenciadores de caso que trabalharam em parceria com as equipes dos serviços.

O objetivo dos agenciadores, que participaram semanalmente de supervisões com a coordenadora geral do projeto, era o de acompanhar o paciente, dedicando-se a criar e/ou fortalecer os vínculos que porventura o paciente pudesse vir a ter, promover novos arranjos possíveis para melhorar e, possivelmente, aumentar a rede social do usuário, favorecendo, dessa forma, a continuidade do cuidado e tendo como referência os focos originais da intervenção criada em Nova Iorque: tratamento psiquiátrico e manejo da medicação; tratamento para abuso de substâncias; treinamento de habilidades da vida diária; prevenção e manejo de problemas de moradia; manejo de dinheiro; e intervenção familiar.

Durante o processo de adaptação do instrumento foi incluída uma área de intervenção que não constava no programa original: violência na comunidade. Cabe ressaltar que tanto essa área quanto a de intervenção familiar, que já constava na intervenção oficial, foram consideradas cruciais para o contexto brasileiro.

\section{Fase 3 - Intervenção para períodos de transição}

Em março de 2010, iniciamos a intervenção (Fase 3) com 20 usuários, indicados pelas equipes dos CAPS entre aqueles, do espectro esquizofrênico, com dificuldades de inserção no tratamento. Foram realizadas entrevistas iniciais com os pacientes que receberam a intervenção e, partir de então, as supervisões foram realizadas semanalmente. Elas eram registradas em ata e gravadas em um aparelho digital. Os agenciadores ficavam incumbidos de levar os formulários preenchidos referentes ao acompanhamento, durante a semana, de todos os pacientes sob sua responsabilidade. Todo o material foi armazenado em pastas identificadas pelo nome do paciente e o CAPS correspondente. Além desta reunião semanal, foram necessárias algumas outras com a coordenadora do projeto e a equipe dos serviços a fim de discutir situações e decisões a respeito dos casos.

A intervenção para períodos de transição foi implantada em três etapas consecutivas: transição, experimentação e transferência do cuidado, tendo, cada uma, duração média de três meses. No início da intervenção e ao final de cada uma das etapas foram aplicados questionários de avaliação da implementação.

Na primeira etapa - Transição - o usuário e o agenciador da CTI-Br formularam um plano de tratamento com duração de nove meses, focado nas áreas identificadas como cruciais para o fortalecimento dos vínculos e favorecimento do engajamento na vida comunitária. A principal tarefa dessa etapa foi a vinculação do sujeito com os recursos comunitários adequados. Nessa fase, os agenciadores da CTI-Br conheceram o paciente e sua família, a sua situação de vida, identificaram quais seriam os focos prioritários para o seu tratamento e o vínculo, elaborando com ele, a família e os seus cuidadores (CAPS, familiares e outros) o plano de trabalho. Ao final desta fase foram realizadas entrevistas, com os instrumentos previamente definidos, com todos os pacientes.

A segunda etapa - Experimentação - correspondeu à implementação do plano terapêutico traçado na fase de transição, que era reavaliado semanalmente nas reuniões de supervisão do projeto. Neste momento, estes sistemas já estavam implantados e em funcionamento e o agenciador da CTI-Br e o usuário podiam verificar in vivo o seu funcionamento e quais pontos precisavam de ajustes. Ao final desta fase, foram realizadas entrevistas, conforme protocolo, em todos os pacientes.

A etapa final - transferência do cuidado - objetivou promover o ajuste da relação construída entre o usuário e a rede de apoio e a saída gradual do agenciador da relação de cuidado estabelecida ao longo dos nove meses. Ao final desta fase, foram realizadas as entrevistas finais, repetindo todos os instrumentos da primeira entrevista, além de uma confraternização de encerramento em cada CAPS, com seus respectivos pacientes, na presença da coordenadora do projeto e das equipes dos serviços.

A partir da leitura dos planos de tratamento e dos formulários preenchidos pelos agenciadores, buscou-se extrair as informações relevantes relacionadas aos focos da intervenção, atendendo aos critérios específicos das fases. Foi construída uma máscara de análise para cada paciente, objetivando organizar e sistematizar os dados obtidos durante os nove meses de agenciamento de caso, com ênfase na evolução e na trajetória do usuário. Esse documento teve como intuito organizar e sistematizar os dados obtidos durante os nove meses de agenciamento de caso dos pacientes que participaram da intervenção para períodos de transição. Cada máscara referese a um determinado paciente. Os dados foram inseridos com base nas informações contidas nos formulários preenchidos (pelos agenciadores e supervisor) no decorrer da intervenção, como já citado anteriormente na adaptação da Fase 2.

Os dados coletados nas entrevistas aos três, seis e nove meses da intervenção, o material áudio-gravado nas reuniões semanais de supervisão dos agenciadores, o caderno de campo do supervisor e o livro ata das supervisões também foram fontes de informação. Essa máscara de análise foi divida em quatro sessões, que se referem às fases da intervenção para cada paciente. São elas: Pré-CTI, Fase 1, Fase 2 e Fase 3.

Para a análise do material, lançamos mão do método descritivo, de modo que os pesquisadores, a partir dos dados 
que constavam nos instrumentos acima mencionados, fizeram uma descrição que sintetizou a evolução da trajetória do usuário durante os nove meses de intervenção, enfatizando os focos da intervenção para cada paciente.

\section{Aspectos éticos}

A pesquisa foi aprovada pelo Comitê de Ética do Instituto de Psiquiatria (IPUB) da UFRJ e pela Comissão Nacional de Ética em Pesquisa do Ministério da Saúde.

Todos os sujeitos forneceram consentimento informado para participação na pesquisa e assinaram o Termo de Consentimento Livre e Esclarecido conforme previsto na declaração de Helsinki e na Resolução 196/96 do Conselho Nacional de Saúde (CNS).

\section{RESULTADOS}

A intervenção propriamente dita (Fase 3) ocorreu entre março e dezembro de 2010. Inicialmente fizemos contatos com as equipes dos serviços a fim de agendar uma ida à reunião de equipe para discussão coletiva dos casos dos usuários que receberiam a intervenção e definição dos focos da intervenção. Cada CAPS escolheu cinco pacientes em consonância com as características dos focos preestabelecidos. Dentre esses, uma usuária saiu da intervenção antes do seu início devido à impossibilidade de contatar esta paciente e seus familiares, e, após quatro meses do início da intervenção, um outro paciente faleceu em decorrência de um câncer de fígado. Seguimos, então, acompanhando os 18 pacientes restantes, juntamente com os agenciadores, até o final da intervenção em supervisão de equipe semanal, conforme relatado anteriormente.

Para melhor visualização dos resultados, a evolução do acompanhamento de cada paciente está descrita sinteticamente de acordo com a etapa correspondente da intervenção nos quadros seguintes, separados pelos respectivos CAPS (Tabelas 1 a 4). Os planos terapêuticos traçados para cada paciente concentraram-se na escolha de duas ou três áreas de intervenção (focos da intervenção).

A fim de ilustrar melhor o contexto de vida dos pacientes acompanhados pela intervenção, apresentaremos dois casos considerados emblemáticos nesse processo. O Sr. César, também conhecido como "Seu César da Árvore", é um homem de aproximadamente 60 anos, pardo e de estatura mediana. $\mathrm{O}$ paciente não tinha contato com seus familiares, vivia em situação de rua - mais precisamente no canteiro de uma grande árvore, próximo ao CAPS - há aproximadamente 20 anos, sem ter qualquer tipo de renda nem documentação. No início do projeto, o CAPS foi acionado pela prefeitura da cidade do Rio de Janeiro para recolher o paciente e conseguir um lugar onde pudesse ficar. Nessa época, o CAPS, que já havia tentado aproximar-se do usuário, retomou o contato. O Sr. César foi selecionado pelo serviço para participar do projeto de pesquisa CTI-Br por se encontrar em situação de vulnerabilidade. Em um primeiro momento, foram identificadas algumas necessidades concretas: a do agenciamento de um local de moradia para o paciente, além da necessidade de solicitação, junto aos órgãos responsáveis, de documentação de identidade (certidão tardia) e benefícios sociais. Também se identificou a necessidade de cuidado (clínico e de saúde mental) e de acesso ao sistema de saúde por meio do tratamento psiquiátrico e clínico. A partir de então, a agenciadora passou encontrá-lo semanalmente, fez contatos frequentes com a equipe do CAPS e conseguiu levar o paciente para uma avaliação clínica no hospital geral de referência da área, apesar da resistência dos profissionais de saúde em receber uma pessoa em situação de rua e com problemas psiquiátricos. Após a realização de exames, diagnosticou-se um câncer de fígado. A agenciadora garantiu o acesso do paciente ao sistema de saúde e a sua continuidade de cuidado. Durante essa fase, ela manteve o acompanhamento ao usuário, que continuava internado no hospital. Após avaliação no Instituto Nacional do Câncer (INCA), o médico contraindicou a cirurgia, não só pelo risco, mas também por se tratar de um caso terminal. A agenciadora seguiu visitando o Sr. César na casa de apoio. A cada dia o seu quadro piorava, com queda do estado geral. Em suas últimas visitas, ela ficou ao lado do paciente, no leito. Conversou com ele e lhe entregou a imagem de Santa Rita. Ambos rezaram enquanto ele fitava a imagem. O usuário pediu que a agenciadora ficasse mais um pouco ao lado dele e revelou que, apesar de saber que estava muito doente, não estava mais sozinho pois agora ele tinha a companhia de uma “amiga”. Uma semana após o seu falecimento, a agenciadora foi, com uma técnica do CAPS, preparar o corpo para, então, prestarem as últimas homenagens ao paciente.

O outro caso é sobre Ciro, um jovem negro, de "20 e poucos" anos, que não sabe ler nem escrever e apresentava-se em más condições de higiene. Apesar de circular sozinho na rua, aparentava ter muitas dificuldades em se autocuidar. Residia com a mãe e o pai em uma casa com precárias condições materiais, sem saneamento básico, em uma comunidade violenta. Os pais são semianalfabetos. A família, que dependia do benefício do usuário para sobreviver, passou por dificuldades financeiras e queixou-se que o benefício de Ciro havia sido suspenso. Nenhum parente se envolvia suficientemente em seu tratamento. $\mathrm{O}$ usuário frequentava o CAPS irregularmente, não tendo se vinculado ao tratamento de forma desejável, além de mostrarse resistente ao uso da medicação. Ciro apresentava alterações comportamentais, geralmente chegava ao CAPS contorcendo o 
Tabela 1. Focos de intervenção da Critical Time Intervention

\begin{tabular}{|c|c|c|c|c|c|}
\hline CAPS & Usuários & $\begin{array}{l}\text { Focos } \\
\text { CTI-Br }\end{array}$ & Anterior à intervenção & Durante a intervenção & Após a intervenção \\
\hline \multirow{5}{*}{1} & $\begin{array}{l}\text { Fabiana } \\
\text { (20 anos) }\end{array}$ & $1 ; 5 ; 6$ & $\begin{array}{l}1 \text { - Em internação psiquiátrica } \\
5 \text { - Acumulava lixo dentro de casa } \\
6 \text { - Frágeis vínculos familiars }\end{array}$ & $\begin{array}{l}\text { Quadro psiquiátrico instável; Situação } \\
\text { de violência; } \\
\text { Maior frequência ao CAPS; } \\
\text { Pai usufruia do benefício da paciente; }\end{array}$ & $\begin{array}{l}\text { Breve internação psiquiátrica; } \\
\text { Maior dedicação da equipe do } \\
\text { CAPS ao caso }\end{array}$ \\
\hline & $\begin{array}{l}\text { Francisco } \\
\text { (53 anos) }\end{array}$ & $5 ; 6 ; 7$ & $\begin{array}{l}5 \text { - Institucionalização de longa } \\
\text { permanência ( } 34 \text { anos) } \\
6 \text { - Sobrecarga familiar } \\
7 \text { - Reside em comunidade violenta }\end{array}$ & $\begin{array}{l}\text { Contratação de um cuidador; } \\
\text { Maior frequência ao CAPS; } \\
\text { Melhora significativa do quadro } \\
\text { psiquiátrico; }\end{array}$ & $\begin{array}{l}\text { Transferência do cuidado } \\
\text { (agenciador-cuidador); } \\
\text { Fortalecimento do vínculo entre } \\
\text { a equipe do CAPS e o cuidador }\end{array}$ \\
\hline & $\begin{array}{l}\text { Fabrício } \\
\text { ( } 37 \text { anos) }\end{array}$ & $6 ; 7$ & $\begin{array}{l}6 \text { - Resistência familiar em ajudar } \\
\text { no cuidado } \\
7 \text { - Violência na comunidade }\end{array}$ & $\begin{array}{l}\text { Ausência de técnico de referência no } \\
\text { CAPS para o caso; } \\
\text { Não tinha condições de ir ao CAPS } \\
\text { desacompanhado }\end{array}$ & $\begin{array}{l}\text { Transferência do cuidado para } \\
\text { um novo técnico de referência }\end{array}$ \\
\hline & $\begin{array}{l}\text { Fernanda } \\
\text { (36 anos) }\end{array}$ & $2 ; 3 ; 7$ & $\begin{array}{l}2 \text { - Abuso de drogas/prostituição } \\
3 \text { - Precárias condições de } \\
\text { moradia/sem documentação } \\
7 \text { - Violência na comunidade }\end{array}$ & $\begin{array}{l}\text { Documentação e benefício social } \\
\text { providenciados; } \\
\text { Vontade de parar o uso de drogas; } \\
\text { Início da medicação injetável }\end{array}$ & $\begin{array}{l}\text { Fortalecimento da rede de } \\
\text { suporte familiar }\end{array}$ \\
\hline & $\begin{array}{l}\text { Fabíola } \\
\text { (68 anos) }\end{array}$ & $5 ; 6$ & $\begin{array}{l}5 \text { - Histórico de múltiplas } \\
\text { internações } \\
6 \text { - Baixa participação familiar no } \\
\text { tratamento }\end{array}$ & $\begin{array}{l}\text { Ausência de técnico de referência no } \\
\text { CAPS para o caso; } \\
\text { Agressões físicas e verbais em família }\end{array}$ & $\begin{array}{l}\text { Fortalecimento do vínculo entre } \\
\text { a equipe do CAPS e a família }\end{array}$ \\
\hline
\end{tabular}

1: Tratamento psiquiátrico e manejo da medicação; 2: Tratamento de abuso de drogas; 3: Manejo de dinheiro; 4: Prevenção e manejo de problemas de moradia; 5: Treinamento de habilidades da vida diária; 6: Intervenção familiar; 7: Violência na comunidade

CAPS: Centro de Atenção Psicossocial; CTI-Br: Critical Time Intervention

Tabela 2. Áreas de intervenção da Critical Time Intervention

\begin{tabular}{|c|c|c|c|c|c|}
\hline CAPS & Usuários & $\begin{array}{l}\text { Focos } \\
\text { CTI-Br }\end{array}$ & Anterior à intervenção & Durante a intervenção & Após a intervenção \\
\hline \multirow{4}{*}{2} & $\begin{array}{l}\text { Élcio } \\
\text { (24 anos) }\end{array}$ & $1 ; 2 ; 7$ & $\begin{array}{l}1 \text { - Não adesão ao tratamento } \\
2 \text { - Abuso de drogas } \\
7 \text { - Violência na comunidade }\end{array}$ & $\begin{array}{l}\text { Quadro psiquiátrico instável; } \\
\text { Inserção no grupo de Narcóticos } \\
\text { Anônimos }\end{array}$ & $\begin{array}{l}\text { Maior frequência ao CAPS; } \\
\text { Aumento do uso regular da } \\
\text { medicação }\end{array}$ \\
\hline & $\begin{array}{l}\text { Eliane } \\
\text { (40 anos) }\end{array}$ & $1 ; 2 ; 6$ & $\begin{array}{l}1 \text { - Não adesão ao tratamento } \\
2 \text { - Abuso de drogas/prostituição } \\
6 \text { - Frágeis vínculos familiares/ } \\
\text { precárias condições de moradia }\end{array}$ & $\begin{array}{l}\text { Situação de violência familiar e na } \\
\text { comunidade; } \\
\text { Providenciados documentação e } \\
\text { benefício social; } \\
\text { Breve internação psiquiátrica }\end{array}$ & $\begin{array}{l}\text { Dificuldades em parar com o } \\
\text { uso de drogas }\end{array}$ \\
\hline & $\begin{array}{l}\text { Ester } \\
(23 \text { anos })\end{array}$ & $3 ; 5 ; 6$ & $\begin{array}{l}3 \text { - Sem benefício/pedia dinheiro na rua } \\
5 \text { - Precariedade no autocuidado } \\
6 \text { - Outro familiar com transtorno mental }\end{array}$ & $\begin{array}{l}\text { Abuso de drogas e em situação de rua; } \\
\text { Internação psiquiátrica }\end{array}$ & $\begin{array}{l}\text { Permaneceu na Fase I até o } \\
\text { final da intervenção }\end{array}$ \\
\hline & $\begin{array}{l}\text { Etelvina } \\
\text { (43 anos) }\end{array}$ & $1 ; 4 ; 6$ & $\begin{array}{l}1 \text { - Não adesão ao tratamento } \\
4 \text { - Precárias condições de moradia } \\
6 \text { - Outro familiar com transtorno mental }\end{array}$ & $\begin{array}{l}\text { Maior frequência em idas ao CAPS; } \\
\text { Providenciado benefício social }\end{array}$ & $\begin{array}{l}\text { Passou a frequentar o CAPS } \\
\text { semanalmente; } \\
\text { Aumento do uso regular da } \\
\text { medicação }\end{array}$ \\
\hline
\end{tabular}

1: Tratamento psiquiátrico e manejo da medicação; 2: Tratamento de abuso de drogas; 3: Manejo de dinheiro; 4: Prevenção e manejo de problemas de moradia; 5: Treinamento de habilidades da vida diária; 6: Intervenção familiar; 7: Violência na comunidade

CAPS: Centro de Atenção Psicossocial; CTI-Br: Critical Time Intervention

pescoço e com sérias contrações musculares, que aparentavam ser devido ao uso de medicação (impregnação). Ele apresentava atitude paranoide, que lhe colocava em risco na comunidade, pois seus delírios envolviam o tráfico, a milícia local e denúncias à polícia. Os familiares continuavam descomprometidos com o tratamento. Eles acreditavam que, com a melhora do filho, poderiam perder o benefício que ganhavam, única fonte de renda para sobrevivência. Por isso, em muitos momentos, deixavam de dar a medicação para o paciente ou diziam para ele não ir ao CAPS. No último mês da intervenção, o usuário apresentou-se mais agitado e nervoso do que de costume, em virtude da situação de violência na comunidade onde morava. A agenciadora chegou a ser abordada por um integrante de um grupo paramilitar para revista, sendo orientada a voltar outro 
Tabela 3. Áreas de intervenção da Critical Time Intervention

\begin{tabular}{|c|c|c|c|c|}
\hline CAPS & Usuários & $\begin{array}{l}\text { Focos } \\
\text { CTI-Br }\end{array}$ & Anterior à intervenção & Durante a intervenção \\
\hline & $\begin{array}{l}\text { Coutinho } \\
\text { (47 anos) }\end{array}$ & $1 ; 5 ; 6$ & $\begin{array}{l}1 \text { - Não adesão ao tratamento } \\
5 \text { - Precariedade no autocuidado } \\
6 \text { - Conflitos familiares - baixa } \\
\text { participação no tratamento }\end{array}$ & $\begin{array}{l}\text { Quadro psiquiátrico instável; Situação } \\
\text { de violência familiar e na comunidade; } \\
\text { Maior frequência em idas ao CAPS; } \\
\text { Tentativas de manejo familiar }\end{array}$ \\
\hline & $\begin{array}{l}\text { César } \\
\text { (60 anos) }\end{array}$ & $1 ; 3 ; 4$ & $\begin{array}{l}1 \text { - Ausência de tratamento de saúde } \\
3 \text { - Precárias condições de vida/miséria } \\
4 \text { - Há } 20 \text { anos em situação de rua }\end{array}$ & $\begin{array}{l}\text { Internação clínica; } \\
\text { Diagnosticado com câncer de fígado; } \\
\text { Falecimento após quatro meses no } \\
\text { hospital }\end{array}$ \\
\hline
\end{tabular}

$3 \begin{aligned} & \text { Carlos } \\ & \text { (51 anos) }\end{aligned} \quad$ 1;3;5 $\quad \begin{aligned} & \text { 1 - Não adesão ao tratamento } \\ & \text { - Ausência de renda/benefício social } \\ & 5 \text { - Precariedade no autocuidado }\end{aligned}$

\begin{tabular}{|c|c|c|}
\hline $\begin{array}{l}\text { Ciro } \\
(20 \text { anos })\end{array}$ & $1 ; 4 ; 7$ & $\begin{array}{l}1 \text { - Uso irregular da medicação } \\
4 \text { - Precárias condições de moradia } \\
7 \text { - Violência na comunidade }\end{array}$ \\
\hline $\begin{array}{l}\text { ecílio } \\
2 \text { anos) }\end{array}$ & $1 ; 5 ; 6$ & $\begin{array}{l}1 \text { - Não adesão ao tratamento } \\
5 \text { - Precariedade no autocuidado } \\
6 \text { - Baixa participação familiar no } \\
\text { tratamento }\end{array}$ \\
\hline
\end{tabular}

Melhora no autocuidado; Maior frequência em idas ao CAPS; Necessidade em adicionar foco em intervenção familiar

Maior frequência em idas ao CAPS; Aumento no uso regular da medicação; Situação de violência familiar

Maior frequência em idas ao CAPS; Aumento no uso regular da medicação; Melhora no autocuidado
Após a intervenção

Breve internação psiquiátrica; Aumento da dedicação da equipe do CAPS ao usuário

$\mathrm{X}$

Breve internação psiquiátrica; Indicação da equipe do CAPS para residência terapêutica (Serviços Residenciais Terapêuticos)

Quadro psiquiátrico instável; Genitora passou a frequentar grupo de família no CAPS

Necessidade em adicionar foco em manejo do dinheiro; Melhora no convívio familiar

1: Tratamento psiquiátrico e manejo da medicação; 2: Tratamento de abuso de drogas; 3: Manejo de dinheiro; 4: Prevenção e manejo de problemas de moradia; 5: Treinamento de habilidades da vida diária; 6: Intervenção familiar; 7: Violência na comunidade

CAPS: Centro de Atenção Psicossocial; CTI-Br: Critical Time Intervention

Tabela 4. Áreas de intervenção da Critical Time Intervention

\begin{tabular}{|c|c|c|c|}
\hline CAPS & Usuários & $\begin{array}{l}\text { Focos } \\
\text { CTI-Br }\end{array}$ & Anterior à intervenção \\
\hline & $\begin{array}{l}\text { Luzia } \\
\text { (29 anos) }\end{array}$ & $1 ; 6$ & $\begin{array}{l}1 \text { - Em internação psiquiátrica há } \\
\text { dois meses } \\
6 \text { - Negligência no cuidado aos dois } \\
\text { filhos }\end{array}$ \\
\hline
\end{tabular}

Leandro
$(40$ anos $)$$\quad \begin{aligned} & 1 ; 3 \text { - Não fazia uso da medicação } \\ & 3 \text { - Sem documentação - vivia com } \\ & \text { benefício da genitora }\end{aligned}$
$4 \begin{array}{ll}\text { Lindalva } \\ \text { (53 anos) }\end{array} \quad \begin{aligned} & 1 ;- \text { Uso irregular da medicação } \\ & 5 \text { - Dependência do marido para ir } \\ & \text { ao CAPS }\end{aligned}$

\begin{tabular}{|c|c|c|}
\hline $\begin{array}{l}\text { Luis } \\
\text { (34 anos) }\end{array}$ & $1 ; 5 ; 6$ & $\begin{array}{l}1 \text { - Não adesão ao tratamento } \\
5 \text { - Precariedade no autocuidado } \\
6 \text { - Alta sobrecarga familiar }\end{array}$ \\
\hline $\begin{array}{l}\text { Lívia } \\
\text { (22 anos) }\end{array}$ & $1 ; 4 ; 7$ & $\begin{array}{l}1 \text { - Não adesão ao tratamento } \\
4 \text { - Precárias condições de moradia } \\
7 \text { - Violência na comunidade e } \\
\text { história de agressão familiar }\end{array}$ \\
\hline
\end{tabular}

\section{Durante a intervenção}

Início da medicação injetável; Maior participação familiar no tratamento;

Relatos de perambulação pelas ruas

Passou a frequentar o CAPS/não aceitava a medicação; Perambulação pelas ruas

Passou a frequentar o CAPS semanalmente;

Maior frequência no uso regular da medicação

Não conseguia sair de casa devido à gravidade do quadro psiquiátrico; Dificuldade da equipe em dedicar-se ao caso

Maior frequência em idas ao CAPS; Maior frequência no uso regular da medicação

\section{Após a intervenção}

Barreira de acesso à moradia devido à violência na comunidade; Necessidade de internação psiquiátrica por duas vezes

Providenciados

documentação e benefício social em esforço conjunto da família e equipe

Continuou a frequentar o CAPS semanalmente; Manteve uso regular da medicação

Internação psiquiátrica; Equipe do CAPS passou a se aproximar mais da genitora

Barreira de acesso à moradia devido à violência na comunidade;

Sem mudanças na situação familiar

1: Tratamento psiquiátrico e manejo da medicação; 2: Tratamento de abuso de drogas; 3: Manejo de dinheiro; 4: Prevenção e manejo de problemas de moradia; 5: Treinamento de habilidades da vida diária; 6: Intervenção familiar; 7: Violência na comunidade

CAPS: Centro de Atenção Psicossocial; CTI-Br: Critical Time Intervention 
dia. Após esse episódio, o usuário reduziu a sua frequência ao CAPS, interrompendo o uso da medicação que fazia no local e deixando de tomar banho. Durante toda a intervenção, a equipe do CAPS mostrou-se presente no acompanhamento do usuário, seja na organização do uso da medicação ou nas atividades diárias de autocuidado, pois o usuário apresentava muitas dificuldades para realizar estas ações cotidianas por conta própria.

Enfim, esses casos têm em comum o baixo grau de instrução/escolaridade, condições socioeconômicas muito precárias, ausência de trabalho/emprego, rede social rarefeita, composta apenas de familiares. A aparência dos usuários denota, na maior parte das vezes, descuido, com dificuldades tanto em relação a aspectos de higiene, quanto de saúde. Há dificuldade na interação social, o que se reflete na inexistência de uma rotina de atividades cotidianas e de relacionamentos. Além disso, há histórias psiquiátricas longas, muitas com diversas internações prévias em instituições psiquiátricas e histórico de tratamento irregular, com dificuldade para tomar a medicação e frequentar o CAPS.

Durante nove meses, acompanhamos esses pacientes, seus familiares e as equipes dos CAPS aos quais eles estavam vinculados. As questões caminharam e alguns pacientes voltaram a frequentar o CAPS com o auxílio dos agenciadores. Outros passaram a tomar a medicação de forma mais regular. E famílias se sentiram mais acompanhadas e voltaram a acreditar que era possível um destino melhor para seus familiares gravemente adoecidos.

É importante frisar que a maior parte dos pacientes vivia em um ambiente extremamente pobre, sem acesso a espaços de lazer ou outras possibilidades de vínculos sociais. Os vínculos eram mantidos apenas com seus familiares e as equipes dos CAPS, e em nenhum dos casos pôde-se contar com a ajuda de agentes comunitários de saúde ou equipes da saúde da família, inexistentes em seus territórios.

O papel do agenciador concentrou-se, então, em favorecer e fortalecer esses dois vínculos com os familiares e com a equipe de tratamento. Em todas as situações em que foi preciso algum tipo de intervenção clínica com os pacientes, a articulação com a rede de tratamento médico geral foi muito difícil e houve necessidade da presença incisiva do agenciador para que o atendimento ocorresse.

Os planos de tratamento foram traçados para que os agenciadores se aliassem aos usuários, suas famílias e às equipes dos CAPS no enfrentamento das condições que, em linhas gerais, determinavam a manutenção desta vulnerabilidade. Os agenciadores acompanharam os pacientes em seus locais de moradia, conviveram com seus familiares, estabeleceram relações com a vizinhança, os visitaram durante períodos de internação, percorreram a rede de serviço social para providenciar documentos de identidade civil e obtenção de benefícios sociais e acompanharam consultas médicas, buscando a resolução de outros problemas de saúde e visando, de maneira ampla, a superação de barreiras que pudessem impedir a melhora do paciente e sua inserção na comunidade.

\section{CONSIDERAÇÕES FINAIS}

Um aspecto comum a todos os pacientes que participaram da intervenção é a constatação da situação de vulnerabilidade a que estavam submetidos. Para alguns autores ${ }^{12}$, a vulnerabilidade é entendida como resultante de um conjunto de aspectos individuais, sociais, coletivos, intersubjetivos, contextuais e relacionados às políticas, práticas e sistemas de saúde e suporte social. Essas dimensões encontram-se entrelaçadas e acarretam maior ou menor suscetibilidade ao adoecimento.

Avalia-se que o sucesso alcançado na adaptação e na implementação da CTI no contexto brasileiro relaciona-se ao processo de trabalho instituído, no qual o agenciador ocupa papel fundamental para o favorecimento ou fortalecimento de vínculos que possibilitem a inserção e a manutenção do usuário na comunidade. Influíram no bom resultado da intervenção, o pequeno número de casos acompanhados por cada agenciador e o contato estabelecido por estes profissionais com os CAPS e outros locais de tratamento, que incluía a participação do agenciador nas reuniões de equipe do serviço.

Estudo realizado no Rio de Janeiro identificou que um dos principais desafios enfrentados pelas equipes dos CAPS é conciliar o cuidado intra-CAPS com o trabalho de acompanhamento dos seus usuários no território ${ }^{13}$. A diversidade de atribuições dos CAPS ${ }^{14}$, as dificuldades inerentes aos transtornos mentais, além do convívio diário das pessoas com problemas urbanos - violência, uso de drogas, desemprego - são, possivelmente, obstáculos concretos para o acesso à saúde e para a continuidade do cuidado às pessoas com transtornos mentais graves no contexto dos serviços comunitários de saúde mental. $\mathrm{E}$, nesse sentido, os agenciadores, ao se aliarem às equipes dos CAPS, constituíram reforço importante no suporte daqueles pacientes.

$\mathrm{Na}$ presente pesquisa, a interação entre pesquisadores-usuários-familiares-profissionais, no contexto da vida cotidiana dos serviços e do território-comunidade, foi um elemento chave para a compreensão da realidade em questão. Os resultados mostram que os problemas e obstáculos relacionados à continuidade/descontinuidade do cuidado não se restringem aos contextos dos serviços, no plano micro, e nem às políticas públicas de saúde mental, em uma análise macrossocial. Ao contrário, relacionam-se às diferentes esferas 
da vida cotidiana, indo para além dos limites dos serviços e demonstrando que as experiências subjetivas relativas à vida e ao processo saúde-doença implicam em aspectos intersubjetivos, institucionais e histórico-culturais que encontram-se entrelaçados e refletem a complexidade do contexto brasileiro.
A violência urbana e as condições de vida, psíquicas, emocionais e cognitivas dos pacientes, além das condições sociais (renda, moradia, escolaridade), também se apresentaram como obstáculos para o acesso aos usuários e aos serviços de saúde, dificultando a continuidade do cuidado.

\section{REFERÊNCIAS}

1. Clément M, Aubé D, Bolduc N, Damasse J, Beaucage C, Tremblay M [Internet]. Canadian Health Services Research Foundation. 2003. [cited 2012 Mar 25]. Available from: http://www.chsrf.ca/Migrated/PDF/ ResearchReports/OGC/clements_e.pdf

2. Adair CE, McDougall GM, Beckie A, Joyce A, Mitton C, Wild CT, Gordon A, Costigan N. History and measurement of continuity of care in mental health services and evidence of its role in outcomes. Psychiatr Serv. 2003;54(10):1351-6.

3. Johnson S, Prosser D, Bindman J, Szmukler G. Continuity of care for the severely mentally ill: concepts and measures. Soc Psychiatry Psychiatr Epidemiol. 1997;32 (3):137-42.

4. Bachrach LL. The challenge of service planning for chronic mental patients. Community Ment Health J. 1986;22(3):170-4.

5. Adair CE, McDougall GM, Mitton CR, Joyce AS, Wild TC, Gordon A, Costigan N, Kowalsky L, Pasmeny G, Beckie A. Continuity of care and health outcomes among persons with severe mental illness. Psychiatr Serv. 2005;56(9): 1061-9.

6. Susser E, Valencia E, Conover S, Felix A, Tsai WY, Wyatt RJ. Preventing recurrent homelessness among mentally ill men: a "critical time" intervention after discharge from a shelter. American J Public Health. 1997; 87(2):256-62.

7. Melo AP, Guimarães MD. Factors associated with psychiatric treatment dropout in a mental. Rev Bras Psiquiatr. 2005;27(2):113-8.

8. Thornicroft G, Tansella M. Quais são os argumentos a favor da atenção comunitária à saúde mental? Pesqui Prát Psicossociais. 2008;3 (1):9-25.
9. Valencia E, Susser E, McQuistion H, Vaccaro JV, Clark Junior GH. Critical time points in the clinical care of homeless mentally ill individuals. In: Vaccaro J, Clark G, editors. Practicing psychiatry in the community: a manual. Washington DC: American Psychiatric Press; 1996. p. 259-76.

10. Valencia E, Susser E, Torres J, Felix A, Conover S. Critical time intervention for homeless mentally ill individuals in transition from Shelter to community living. In: Breakey W, Thompson J, editors. Mentally ill and homeless: special programs for special needs. Amsterdam: Harwood Academic Publishers; 1997. p. 75-94.

11. Valencia E, Van Hemert AM, Van Hoeken D, Van der Plas A. Gestructureerde zorgtoeleiding met critical time intervention. Sociale Psychiatrie. 2006;78:41-7.

12. Ayres JRCM, Calazans GJ, Saletti Filho HC, França-Júnior I. Risco, vulnerabilidade e práticas de prevenção e promoção da saúde. In: Campos GWS, Minayo MCS, Akerman M, Drumond Júnior M, Carvalho YM, organizadores. Tratado de Saúde Coletiva. Rio de Janeiro: Fiocruz; 2009. p. $375-419$.

13. Cavalcanti MT, Dahl CM, Carvalho MCA, Valencia E. Critérios de admissão e continuidade de cuidados em centros de atenção psicossocial, Rio de Janeiro, RJ. Rev. Saúde Pública. 2009;43(1):23-8.

14. Leal EM, Delgado PGG. Clínica e cotidiano: o CAPS como dispositivo de desinstitucionalização. In: Pinheiro R, Guljor AP, Gomes A, Mattos RM, organizadores. Desinstitucionalização na saúde mental: contribuições para estudos avaliativos. Rio de Janeiro: CEPESC, IMS/LAPPIS, ABRASCO; 2007. p. 137-54.

Recebido em: 03/05/2012 Aprovado em: 18/05/2012 